\title{
Estrategias de la comunicación educativa en las tic, como alternativa de apoyo en el contexto universitario ecuatoriano
}

DOI: $10.46932 / \mathrm{sfjdv} 2 \mathrm{n} 2-152$

Received in: March 1st, 2021

Accepted in: May 30th, 2021

Gricelia Lorena Mendoza Alcívar

Ingeniera Comercial

Master universitario en Tecnología Educativa y Competencias Digitales

Docente en Nivelacion de Carrera

E-mail: miangeljossue@gmail.com; gricelia.mendoza@utm.edu.ec

\section{Gustavo Adolfo Santana Sardi}

Licenciado en Ciencias de la Educación Técnica mención Contabilidad Computarizada

Diplomado en Desarrollo de Estrategias Digitales de Aprendizaje

Magister en Gerencia Educativa

Analista del Centro de Promoción y Apoyo al Ingreso

Universidad Técnica de Manabí

E-mail: gusansar@hotmail.com, gusansar@gmail.com,gustavo.santana@utm.edu.ec

Francisco Antonio Mawyin Cevallos

Licenciado en Ciencias de la Educación especialidad Psicología y Orientación Vocacional

Magister en Orientación Educativa Vocacional y Profesional

Doctor Dentro del Programa de Doctorado en Educación

Docente Auxiliar Tiempo Completo

Universidad Técnica de Manabí

E-mail: franciscomawyin2016@gmail.com; francisco.mawyin@utm.edu.ec

\section{Kerly Leomar Toala Vera}

Licenciada en Ciencias de la Educación especialización Historia y Geografía

Magister en Docencia e Investigación Educativa

Analista del Centro de Promoción y Apoyo al Ingreso

Universidad Técnica de Manabí

E-mail: kerly.toala@utm.edu.ec; kerlyta13@hotmail.es

\section{RESUMEN}

El presente trabajo tiene como objetivo investigar las estrategias que se pueden emplear durante la comunicación educativa usando las tecnologías de información y comunicación, con la finalidad de que sea una alternativa de apoyo para los docentes y mejoren sus métodos de enseñanza, esta investigación se realizó a través de la profundización documental, teniendo en cuenta las palabras con mayor relevancia que cada autor menciona. Siendo la comunicación la principal manera de compartir información inmerso en la sociedad, se lo ha usado de manera universal para la práctica enseñanza-aprendizaje en general, esto se lo conoce como comunicación educativa, con los continuos avances tecnológicos las entes educativas se han visto obligados a actualizarse continuamente y aprovechar al máximo los recursos tecnológicos actuales, estos recursos son conocidos como las Tecnologías de Información y Comunicación (TIC), estos son diversas herramientas que han influido enormemente en la forma de comunicarse actualmente. En Ecuador la gran mayoría de universidades están optando por las TIC como un recurso indispensable en el transcurso de cada una de las asignaturas, facilitando varias situaciones que de manera presencial llega a 
ser un problema al realizarse, todo esto respondido en diversas estadísticas realizadas por varios autores en los últimos años. Esta investigación se realizó bajo la técnica documental tipo bibliográfico junto con el análisis teórico-metodológico de diversos artículos y tesis, permitiendo averiguar de una mejor manera sobre las estrategias que el docente puede emplear durante la comunicación educativa usando las TIC en el contexto universitario ecuatoriano.

Palabras claves: comunicación, educación, TIC, estrategia, plataformas virtuales.

\section{INTRODUCCIÓN}

La comunicación es uno de los pilares más importantes de una sociedad, en palabras del autor Obregón (2007) señala que:

La comunicación contribuye a la reflexión sobre lo que las comunidades o sociedades creen qué es el desarrollo, facilita entornos para que se determinen las metas y estrategias, permite analizar las realidades de los pueblos y establecer políticas a seguir, y se convierte en una herramienta clave para promover procesos de cambio a nivel político, social, comunitario e individual. (p.1).

Donde se confirma el cómo un grupo grande de personas pueden llegar a compartir sus ideas y reflexionar entre todos gracias a la comunicación, y con esto, pensar en cómo poder mejorar el entorno en general de toda la comunidad y establecer un objetivo que los beneficiara a futuro. El uso de la comunicación es indispensable al momento de transmitir alguna información o alguna idea hacia una o varias personas, uno de los entornos en donde esto es importante es en el ámbito educativo, donde el emisor o el que transmite el mensaje es el docente, y este se encarga de compartir su conocimiento e información a los receptores o estudiantes, así, la constante comunicación entre estos dos actores contribuye sin lugar a dudas al desarrollo de la personalidad de los estudiantes y a la propia construcción del conocimiento. Es esta la razón por la que en el mundo contemporáneo comienzan a hacerse frecuentes nuevos modelos educativos que superan las tradicionales formas de enseñar y aprender, y que apuestan por el diálogo creativo y participativo. (Mayre Barceló Hidalgo et al., 2016, p.2). Comúnmente se opta por enseñar a los estudiantes únicamente de forma presencial, con los instrumentos de educación como son libros de texto, artículos científicos y ejercicios que sirven como un ejemplo práctico sobre lo que se enseña, el deber del estudiante es atender a toda información que reciba del docente, adquirirla y entenderla, esto mejorara su percepción en las cosas y podrá procesar mejores ideas cada vez que conozca algo nuevo del mundo que le rodea.

A lo largo de los años la manera en que el mundo se comunica ha estado evolucionando constantemente partiendo desde el hablar frente a otra persona, pasando por el intercambio de mensajes a media distancia como lo fueron las señales de humo en los comienzos de la historia del hombre, entre más pasaban los años la sociedad buscaba mejores maneras de comunicarse, llegando a utilizar hasta animales para mandar y recibir mensajes, un ejemplo de esto fue el uso de palomas entrenadas, también se crearon 
varios aparatos tecnológicos para el intercambio de comunicación, hasta la actualidad en donde se llegan a utilizar los conocidos smartphones y el uso de las tecnologías de información y comunicación, conocidas comúnmente como TIC. Junto a los agigantados pasos de la evolución tecnológica, esta ha traído consigo varias maneras para comunicarse, alcanzando el punto de saber cómo se encuentra una persona que está al otro lado del mundo, e incluso cuales son las ultimas noticias que han sucedido en un lugar en concreto, todo esto gracias a la existencia de las TIC, las Tecnologías de la Información y Comunicación (TIC) remontan su origen a la aparición de las computadoras y el internet que conllevo a la facilidad del acceso de información, dando lugar a la creciente e importancia sociedad tecnológica. De esta forma, la revolución tecnológica ha marcado un antes y un después en la sociedad mundial (Hernández et al., 2018, p.674), esto repercuto en como ahora una persona adquiere nuevo conocimiento con la facilidad y rapidez de un solo click, así mismo el establecer relaciones amistosas con otras personas, incluso con aquellas que son de otras partes del mundo, se resumió en sencillos mensajes enviados una aplicación de mensajería instantánea. Espinoza Freire et al., (2018) aclara que “estas tecnologías poseen una de las herramientas más importantes que constituye un hito tecnológico de gran impacto social, Internet; a través de sus recursos se tiene acceso a información de forma rápida, sencilla, eficiente y de calidad." (Espinoza Freire et al., 2018, p.13),siendo sus palabras llenas razón debido a como el Internet a rompido diversas barreras que frenaban el avance de una persona, gracias a este, el poder encontrar información sobre cualquier cosa que haya pasado en cualquier lugar del mundo se ha vuelto tan fácil como hacer click en una página web.

Ecuador mostraba signos de bajo uso de diversas herramientas tecnológicas como son las TIC e infraestructuras de comunicación, afectando en el desarrollo general del país, tanto en el ámbito laboral, educativo, social, entre otros. Vinueza (2017) indico que la poblacional ecuatoriana ha tenido un mejor acceso a internet, señalando que desde el 2008 hasta el 2016 más de la mitad de los ecuatorianos tenían acceso a internet, pero aun así es complicado llegar a ciertas zonas del país por la falta de infraestructura y niveles tecnológicos que el Ecuador posee con respecto de las TIC (p.361). Así también, Ecuador en los últimos años logro mejorar todo tema de desarrollo de tecnología y servicios, como por ejemplo duplicar la penetración y velocidad de Banda Ancha Fija y de Banda Ancha Móvil, motivando a que las personas busquen y sigan nuevas tendencias de mercado y de estudio para temas tecnológicos, con la finalidad de que el progreso se haga de manera colectiva y no solamente en ciertas áreas. (Falconí Asanza, 2017, p.84). El autor Felipe et al., (2017) señala que:

"Las nuevas tecnologías proveen instrumentos para mejorar los procesos de enseñanza aprendizaje dentro de los centros educativos. Proporcionan la colaboración entre la comunidad educativa; que incluye a docentes, directivos, estudiantes y padres de familia. A través de las distintas herramientas se puede lograr la formación que requieren los habitantes para estar acorde al mundo laboral; y a su vez contribuyen a culminar las desigualdades que se dan en la sociedad.” (p.71). 
Aquí se demuestra como estas herramientas harán un gran cambio para los estudiantes que al terminar la universidad estén en búsqueda de un trabajo, es muy común el usar herramientas TIC en la gran mayoría de trabajos encontrados en el país, tal así que se ha vuelto una obligación que la persona tenga conocimientos acerca de los recursos tecnológicos y las TIC, con esto se aumenta la probabilidad de que el estudiante llegue a conseguir un puesto en el trabajo que desea. Si se habla en el contexto educativo, las TIC presentan grandes oportunidades tanto para los docentes como, y sobre todo, para los estudiantes, en términos de accesibilidad, de flexibilidad y, en algunos casos, de costos, por ende, la necesidad de redefinir los modelos de enseñanza tradicionales permitirán que los procesos de aprendizajes lleguen a ser más flexibles, dando las posibilidades de acceder a la información impartida por el docente de manera más sencilla y con la probabilidad de tener un mejor entendimiento, según el autor Salinas (2004) el impacto del uso de las TIC se mostrara en tres aspectos críticos los cuales son: "la necesidad de proporcionar acceso a una cantidad cada vez mayor a la educación postsecundaria, en la necesidad de modalidades cada vez más flexibles en términos de lugar, espacio, ritmo, itinerarios, etc.... y en la importancia que va tomando la financiación y, en consecuencia, los costos de la educación superior." (pp. 1-2). Aun así, existen varias razones por las cuales a los docentes les cuesta adaptarse a las oportunidades que ofrecen las tecnologías de la información y la comunicación (TIC), algunas de ellas son el que los estudiantes que llegan a la universidad aún esperan clases tradicionales, teniendo la ilusión de que los nuevos métodos implican mayor esfuerzo, los procesos de evaluación que varias instituciones tienen diseñadas y son aplicadas por los docentes a los estudiantes no favorecen ni reconocen la innovación con las TIC, los programas o currículos escritos de la institución reflejan métodos de enseñanza tradicional, entre otros. (Zempoalteca Durán et al., 2017, p.82), estas y muchas otras razones frenan el deseo de llevar a cabo el uso de estas tecnologías, aunque a lo largo de los últimos años el país Ecuador ha mostrado un significante avance en lo que es el uso de estas tecnologías en el ámbito educativo, aun así, queda un largo camino para que toda institución de educación superior establecida en el país aproveche en su plenitud las TIC y sus diversas estrategias. El presente artículo tiene como propósito realizar un análisis teóricometodológico acerca de las estrategias que existen en la comunicación educativa usando como apoyo las tecnologías de información y comunicación, y como estas pueden influir al contexto universitario en Ecuador.

\section{MATERIALES Y METODOS}

El estudio analítico realizado, basado principalmente en la profundización documental, en base a la investigación realizada acerca de las estrategias que existen en la comunicación educativa haciendo uso de las TIC como apoyo, se tuvieron en cuenta elementos de mayor relevancia desde el punto de vista de 
los autores y se ofrecieron diversas consideraciones a quienes van dirigido el presente documento. La generación del trabajo documental, se decidió por realizar el uso de diversos métodos de naturaleza teórica en la investigación científica, los cuales fueron: histórico - lógico; analítico - sintético; hermenéutico; deductivo y de carácter empírico, el análisis de documentos.

\section{RESULTADOS}

El uso constante de material tecnológico es actualmente indispensable en la vida diaria de la sociedad, tal como lo explica Lenin Zambrano-Zambrano et al., (2021):

Las Tecnologías de la Información y la Comunicación son un elemento esencial en los nuevos contextos y espacios de interacción entre los individuos. Estos nuevos espacios y escenarios sociales conllevan rasgos diversos que generan la necesidad del análisis y reflexión en torno a sus características.(p.982).

Agregando lo dicho anteriormente, AGUIAR et al., (2019) menciona:

Se crean nuevos ambientes de desarrollo donde surgen interrogantes y se ofrecen variadas respuestas no solo en la teoría, sino en la práctica, utilizando como complemento de salida el empleo de las tecnologías en respuesta a un pensamiento creativo, innovador y propuestas pertinentes para esta época. (p.13).

Entrando al entorno educacional, se está comenzando a utilizar estas tecnologías tanto para la transmisión de información que el docente transmite como clase, así como también en la presentación de trabajos que los estudiantes entregan a los profesores. Como se menciona anteriormente, estos nuevos espacios o ambientes de desarrollo crean un espacio donde los individuos, en este caso el docente y los estudiantes, puedan afrontar entre si todas aquellas interacciones que normalmente ocurren de manera presencial, e incluso mejorando el tiempo de respuesta y el análisis de estas en diferentes puntos de vista, complementando así cada solución que se llega a encontrar según pasa el tiempo. Tal como lo menciona Felipe et al., (2017), las TIC se han convertido en:

... pieza clave de los avances y cambios en el ámbito educativo que se viven en la actualidad, en especial en la educación superior, al estar inmersa en el ambiente universitario y en la docencia, que cada día va tomando mayor importancia por lo que aporta en el desarrollo económico y social de los pueblos. (pp.69-70).

Ayudando así que la educación tenga un mayor alcance a varios sitios donde se les complique completar los estudios de manera presencial, llegando al punto de terminar completamente una carrera entera de manera virtual solo usando las TIC. Al comenzar a usar las TIC en una institución de educación superior, los docentes deben realizar continuos cambios en las estrategias didácticas, así también en los sistemas de comunicación y distribución de los materiales de aprendizaje, enfatizando la disponibilidad y 
las potencialidades de las tecnologías. Con esto, es necesario tener el conocimiento acerca de la responsabilidad de los recursos de las TIC para la docencia realizando una planificación y configuración pedagógica acerca de estas tecnologías, igualmente se recibe soporte de los servicios de informática de la universidad o el propio soporte técnico de la herramienta que se llegara a usar. (Domínguez Dias, 2016, p.159).

Las TIC ofrece a los docentes una amplia y variada información que les permiten mantenerse actualizados, facilitando los procesos de enseñanza-aprendizaje e investigativos, estos permiten acoplarse a la comunidad pedagógica para el intercambio con otros docentes sobre diversos temas del ámbito educacional, facilitando su constante capacitación y superación comenzando un cambio a las prácticas rutinarias que realiza. (Espinoza Freire et al., 2018, p.14). Para aquellos que están envueltos en la enseñanza, es indispensable estar siempre atentos a las ultimas noticias acerca de tecnologías, en especial aquellas que puedan influir positivamente durante el proceso de enseñanza-aprendizaje, así, el docente debe mostrar un interés por el avance y la actualización de métodos para compartir información de manera mas entendible y sencilla a los estudiantes. Existen diversas herramientas que los docentes pueden usar para mejorar el desarrollo de un aprendizaje significativo en el entorno del estudiante, en el estudio realizado por Felipe et al., (2017) acerca del uso de las TIC en diversas universidades del Ecuador se llegó a averiguar qué herramientas son mayormente usadas para el proceso de enseñanza en donde las redes sociales son las más usadas con un $17,7 \%$ seguido del correo web con 15,8\% y los software de presentación con un 12,8\%. Llegando a la conclusión que los docentes ven más factible usar las redes sociales debido al frecuente uso que tiene los estudiantes con estos, y la facilidad que tienen para comunicarse entre sí (pp.73-74). El correo web como segundo más usado, mayormente para comunicar algo en concreto y de forma detallada, y como tercero lo que son los softwares de presentación, usadas durante las clases para transmitir de forma sencilla y fácil de entender a los estudiantes. Además de las herramientas anteriormente ya mencionadas, existen otras que actualmente se han vuelto mas populares y llegan a facilitar diversas situaciones para ambos actores. Dicho esto, las herramientas mas importantes son:

- Elementos e-learning, los cuales son recursos educativos que se encuentran en la nube, estos elementos permiten realizar el proceso de enseñanza-aprendizaje de forma sincrónica o asincrónica, teniendo cada uno de estos sus ventajas, en las asincrónicas permiten realizar estos procesos en distintos usos horarios, dando la posibilidad al estudiante de completarlos durante un rango de tiempo definido. (Vinueza et al., 2017, p.363). Según Huaman-Huayllapuma (2019) señala que "se puede implantar a todas las modalidades pedagógicas: la formación presencial, la formación a distancia síncrona o asíncrona, y a toda clase de técnicas de formación (expositiva, 
participativa, autorreactiva, tutorizada, etc.).” (p.12). Así, para implementar e-learning, el docente necesita informarse sobre todas las herramientas que le pueden ayudar a mejorar su enseñanza, desarrollando una plataforma con diversos recursos didácticos según su plan de trabajo, e incluso si es necesario, la misma plataforma puede configurarse para la implementación de mas docentes que deseen usarla también. Las plataformas virtuales ayudan a los estudiantes a fortalecer los conocimientos aprendidos en clase utilizando recursos online, esto permite estar conectados los estudiantes y el docente en un mismo sitio compartiendo ideas, información, analizando y resolviendo dudas de algún tema.

Las e-learning cuentan con varias características positivas como por ejemplo el aprendizaje basado en web, en donde permite al estudiante leer la información, analizar ideas, realizar un resumen y exponer durante la clase todo lo que llego a aprender, mientras que el docente puede realizar una retroalimentación basada en las dudas e ideas que tienen los estudiantes. Otra característica positiva de las e-learning es la posibilidad de comunicación de manera semipresencial con el docente en cualquier momento y en cualquier parte siempre y cuando tenga una conexión a internet, esto ayudara al momento en que uno o varios estudiantes tenga un problema y necesiten una tutoría, el docente puede fácilmente establecer foros donde se discutan aquellos problemas y quede toda la información hablada para que puedan revisarlas en cualquier momento que necesiten.

Los enfoques de e-learning pueden combinar diferentes tipos de componentes del propio elearning entre ellos están sus contenidos, en donde se contaran recursos informativos como pdf, presentaciones en power point o investigaciones en Word, todo esto para que el estudiante tenga una guía sobre los trabajos que necesita realizar, aquí también se agregan los videos de refuerzos acerca de un tema en específico, también se encuentra las tres e-learning del docente: E-coaching, e-mentoring y e-tutoring las cuales son el asesoramiento, aprendizaje y tutoría, respectivamente, dada al estudiante en tiempo real a través de una plataforma virtual. El aprendizaje colaborativo en las e-learning es algo indispensable, esto se origina estableciendo grupos online en donde los estudiantes pueden debatir, opinar e intercambiar ideas entre sí, incrementando la confianza entre ellos y conociendo diferentes puntos de vista de un mismo tema. Y, por último, el recurso más usado actualmente, el aula virtual, establecidas en páginas dedicadas a la creación de estas, las aulas virtuales es un método de enseñanza sumamente efectivo en donde se emplea la combinación de recursos multimedia e interactiva para que la clase sea más agradable e interesante para los estudiantes. (Palacios Gordillo \& Vergara Tejada, 2017, p.16-19).

Todas estas herramientas favorecen con diversas características al ámbito educacional, como la facilidad al acceso de cualquier información mediante una red conectada al internet, la posibilidad de 
participación de cualquier persona que este en otro lugar del mundo, permitir el consumo de productos audiovisuales y multimedios sean videos explicativos o las propias noticias sucedidas en una zona lejana, y también el poder consultar una gran cantidad de información en muy poco tiempo, a diferencia de años anteriores donde se hacia un esfuerzo y gran cantidad de tiempo para poder encontrar un fragmento de la información que se necesitaba.

- Sitios web, son entornos basados en código HTML, los cuales permiten optimizar las herramientas web que sirven para desarrollar productos y servicios Online según las diferentes necesidades que tiene un grupo de personas. Definiéndolo mejor, un sitio web, es el conjunto de páginas web, en una página web se encuentra texto o gráficos multimedia centrados en un tema en particular. El acceso a la información se ha vuelto increíblemente sencilla gracias a la creación de la World Wide Web. En un sitio web, la página principal constituye la primera página a la que se accede y en la cual se centran los usuarios. La página principal se ha convertido en la carta de presentación de cualquier institución Dependiendo de la facilidad para acceder a la información de la página web principal, generalmente los usuarios toman la decisión de continuar o no navegando por dichos sitios web. (Acosta \& Luján-Mora, 2017, p.47). Con esto dicho, las instituciones de educación superior optaron por desarrollar sus propios sitios web, teniendo un elemento mas para hacerse conocer, esta vez de manera mundial.

El nacimiento de la WWW, dio la posibilidad de que cualquier persona que tuviese acceso a internet podría entrar a cualquier página web que quisiera, sin importar en donde se encuentre. Actualmente existen diversos programas y herramientas que hacen del desarrollo de sitios web mas sencillo, hasta el punto de que personas con un conocimiento básico en computación pueden crear un sitio web propio, cabe destacar que en este caso esas páginas web creadas son de manera local, es decir, solo pueden acceder a ella aquellas personas que estén conectadas en una misma señal de internet.

La existencia de las páginas educativas ha ayudado de gran manera tanto a docentes como estudiantes, aunque estas mismas presentan ventajas como la rapidez en encontrar alguna información al interior de ella en lugar de tener que buscar en alguna enciclopedia, otorgan soporte en línea para cualquier problema que se presente y obtener de ellos una solución. En cambio, la falta de control sobre la información que se sube deja riendas sueltas a personas que quieran modificarla para buscar algún beneficio propio, normalmente se ve esto en páginas webs comunitarias como por ejemplo Wikipedia, además si no se tiene el suficiente conocimiento de ciberseguridad, toda información subida y guardada en algún servidor quedaría vulnerable ante aquellos que viven del robo de datos. 
Las plataformas virtuales especializadas en la educación permiten crear, gestionar y distribuir las actividades formativas virtual a través de un servidor web, estas proveen funciones que son de gran utilidad para la gestión del aprendizaje como lo son la distribución de recursos y actividades, la gestión de usuarios posibilitando la asignación de un rol para cada uno, el control de acceso donde se puede configurar para solo tengan acceso los estudiantes con un correo único dado por la institución de educación superior, seguimiento, evaluaciones, servicios como foros, entre muchos otros. Todo esto con el objetivo de brindar una educación a distancia con una constante interacción entre docente - estudiante.

Existen diversas plataformas virtuales que han sido reconocidas por varias instituciones de educación, mayormente empleadas por aquellas de educación superior, debido a su facilidad al configurarse y por sus diversas funciones que estos contienen, uno de los más usados, confiables y gratuitos es la plataforma Moodle el cual, según palabras de Río et al., (2018):

\begin{abstract}
"Moodle es una herramienta flexible que promueve el trabajo colaborativo y los procesos formativos, que despierta el interés de los estudiantes hacia las tecnologías y que se alinea con los intereses de una población nativa digital. Esta herramienta ha demostrado ... que mejora la participación en el aula y el rendimiento académico de los estudiantes" (p.180).
\end{abstract}

Esta herramienta se puede llegar a usar tanto en asignaturas presenciales como virtuales, ya que facilita el intercambio de información, sea noticias, cuestionarios, tareas, entre otros. También facilita el seguimiento de clases o actividades para los estudiantes que no tienen la forma de asistir de manera presencial a aquellas clases por diversas razones, e incluso también permite adaptarse según la necesidad y el ritmo de aprendizaje que tenga el estudiante ofreciendo diversos formatos, estos pueden ser auditivos, visuales, audiovisuales o escritos. Así, Moodle cuenta con diversos módulos que puede usar el docente, estos son:

- Tareas, junto con las calificaciones y comentarios correspondientes, donde los estudiantes pueden subir sus archivos con la fecha respectiva al servidor, así como recibir notificaciones.

- Consulta o votación, para saber la opinión o la estadística de algún tema.

- Foro, exclusivo para docentes y cursos abiertos. Pueden existir varios temas de discusión.

- Diario, el cual contiene la información privada entre docente y estudiante, también en el formulario puede haber una pregunta abierta que puede ser evaluada.

- Cuestionario, que es una base de datos que el docente puede alimentar continuamente o simplemente emplear. Los cuestionarios se califican automáticamente y las preguntas tienen la opción de mostrarse aleatoriamente para evitar la copia. 
- Recursos, admite diversos contenidos digitales, como Word, PowerPoint, Excel, Flash, vídeo o sonidos, y pueden enlazar aplicaciones, transferir datos, etc.

- Encuesta, instrumento para la medición de resultados de las clases en línea. Este puede generar estadísticas y gráficos. (Fernando Martínez-Sarmiento et al., 2019, pp.483484).

Estos módulos encontrados en la plataforma Moodle ayudan y complementan enormemente en las actividades que el docente puede realizar con los estudiantes.

Así también, se encuentra "Google suite for education", la cual es un paquete de herramientas gratuita hecha completamente por la empresa Google, en donde según la investigación realizada por Haro ( 2020), "Google for education es una plataforma innovadora dentro del aula tanto para el profesorado como para el alumnado, la cual sirve para trabajar y aprender con las nuevas tecnologías a la par que son herramientas seguras y gratuitas" (p.7), en este paquete se encuentran diversas herramientas con funciones que ayudan enormemente tanto al docente como al estúdiate, además de que cuenta con una gran variedad de configuraciones, que se adaptan a un grupo en cuestión. "Google suite for education" provee cinco herramientas que servirán como soporte a los estudiantes en la realización de sus trabajos, como a los docentes en la organización de actividades y revisión de tareas. Gmail permite tener una dirección de correo con la extensión utilizada en el centro educativo, tomando como ejemplo [nombre]@[extencion_institucion], estas direcciones de correo la proveen los administradores y solo podrán ser utilizados hasta que el alumno o docente deje de pertenecer al centro institucional. Esta herramienta es mayormente usada para la comunicación e intercambio de información mayormente importante, así también como para la creación de cuentas exclusivas de sitios web educacionales, así también la existencia de estos correos ayudara al docente para poder organizarse con sus respectivos alumnados y no tener que buscarlos de uno a uno de forma presencial. También son usados para la cuando se necesitan compartir archivos o carpetas virtuales que el docente genere y desee que sus alumnos puedan interactuar con ellas, para esto se usara la herramienta llamada Google Drive, esta permite la administración de archivos creados o subidos en la nube dando una interfaz sencilla y fácil de entender para toda persona, ayuda también al generar una estructura de carpetas y subcarpetas para la organización de temas, y de igual manera estas pueden ser compartidos de manera individual o grupal según la necesidad. También se encuentran editores de documentos online, sean de texto, hojas de calculo o presentaciones de diapositivas, estas herramientas no solo permite crearlos, también deja modificar archivos que se hayan subido a la nube y se encuentre en Google Drive, sin importar que versión se haya usado, Google Docs es compatible con ellos y se adapta al formato que se haya usado en el archivo, este provee una cinta de opciones donde se puede modificar a gusto la información encontrada en el 
documento, esta herramienta también permite compartir los documentos creados con otras personas y la posibilidad de modificar el documento en tiempo real entre dos o más usuarios. Google Calendar es una herramienta en donde se puede agendar cualquier tipo de evento que sea relativamente importante, esta herramienta trae una interfaz sencilla de entender, además de que acopla toda fecha que sea importante según el país en donde se creo la cuenta. Cualquier evento que se haya ingresado manualmente será avisado minutos antes de la hora que se estableció en la información de dicho evento, muy útil para aquellos que no tienen una muy buena memoria para recordar una gran cantidad de situaciones importantes. (Cortes, 2017, p.81-83). Además de ofrecer estas herramientas, Google posee otras que se pueden usar en cualquier ámbito, un ejemplo es Google Meet el cual es una herramienta donde se pueden realizar videoconferencias siempre y cuando se tenga una cuenta de Google, sea personal o institucional, también deja la opción de que solo se unan a la videoconferencia aquellos usuarios con un mismo tipo de cuenta, mayormente usada para diferenciar aquellas personas que no pertenecen a la institución que esta realizándola. Varias de sus opciones incluyen el poder grabar en cualquier momento la reunión en la que se este presente y una vez concluida el video se guardara en una carpeta especifica encontrada en el interior de la herramienta Google Drive, también incluye el ubicar una pizarra en donde cualquiera que esté en la reunión pueda interactuar con ella, sea escribiendo, dibujando o señalando algo, esto con el fin de volver mas interactiva las reuniones y no ser completamente lineales. Es interesante saber que todas las herramientas habladas anteriormente pueden llegar a utilizarse, no solo en un equipo de cómputo, sino también en cualquier dispositivo móvil o smartphone, siempre y cuando tenga acceso a internet. Si se habla de una herramienta de Google desarrollada exclusivamente para la educación existe Google ClassRoom, según palabras de Tarango et al. (2019) esta hace “... posible que cualquier usuario con cuenta de Gmail pueda unirse a clases existentes o crear las suyas propias.“(p.94), también menciona que su carácter gratuito y sin fines publicitarios hace de esta herramienta algo especial a diferencia de otros productos de Google. Con una página principal sencilla donde se muestran las clases en donde el usuario esta ingresado y las paginas de anuncios y actividades de curso mas un calendario donde señala las fechas de inicio y limite de cualquier trabajo en una clase vuelve este sitio web sencillo de entender y con una cantidad mínima de objetos en la pantalla, esto para no molestar mucho al usuario, además todo material que sea utilizado en la plataforma se guardara automáticamente en el Google Drive del propietario, esto sirve como un respaldo a todo documento subido haciendo que los estudiantes y profesores no se preocupen por estos. Esta plataforma virtual también se integra con los otros productos de Google como lo son Google Drive, Docs, Calendar y Gmail. (Tarango et al., 2019, p.95). Es necesario tener en cuenta que emplear las plataformas web como un recurso único durante el periodo del estudiante en la asignatura lo obligara a tener que aprender por sí mismo, esto es conocido como autoaprendizaje. 
- Repositorios, son archivadores digitales y temáticos de las diferentes áreas del conocimiento, estos se encuentran organizados en diferentes bases de datos, las cuales funcionan a través de un proceso de indexación. Esto permite que el estudiante acceda de forma instantánea y dinámica a la información en cualquier lugar del mundo. (Vinueza, 2017, p.363). Bustos González \& Fernández Porcel (2020) definen al repositorio como aquella que “... contiene mecanismos para importar, identificar, almacenar, preservar, recuperar y exportar un conjunto de objetos digitales, normalmente desde un portal web. Esos objetos son descritos mediante etiquetas o metadatos que facilitan su recuperación.” (p.9), explicado de una manera mas directa, estos repositorios contienen varios tipos de información que han sido subidos en el trascurso del tiempo, cualquiera de estos se pueden recuperar para su uso, varias universidades tienen sus propios repositorios de información, en los cuales incluyen la gran parte de libros que se encuentran disponibles en la biblioteca universitaria, esto para que cualquier estudiante perteneciente a la institución pueda acceder a ella sin problema y en cualquier momento, también son usadas para el desarrollo de syllabus, ya que este se vuelve más sencillo de desarrollar para los docentes.

Todas las TIC que se mencionaron son de las más usadas por las instituciones de educación superior debido a su fácil accesibilidad y sencillo manejo, en donde hasta las personas menos adentradas en la tecnología pueden usarla.

Como se habló anteriormente, junto a la llegada de las TIC y su evolución constante, las universidades comenzaron a optar por la educación a distancia, en donde, según la investigación realizada por Mendieta \& García Mendieta (2018) señala que "La educación a distancia se basa en contexto pedagógico y didáctico, mediado entre profesor y el estudiante, quiénes se encuentran ubicados en espacios diferentes, estimulando el aprendizaje de forma independiente y también la colaboración entre pares.” (p.129), en el contexto universitario, se comenzó la idea de desarrollar una posibilidad para que futuros estudiantes puedan asistir a las clases de su carrera sin tener que ir a la institución. De ahí se optó a la creación de carreras virtuales, donde una persona puede ver y aprender todo lo necesario para terminar una carrera universitaria sin tener que estar de manera presencial con el docente, esto también da la ventaja de acceder en cualquier momento a toda la información que los docentes proveen e incluso la posibilidad de que el profesor pueda compartir una clase completa con sus estudiantes a través de una plataforma de videoconferencia como si estuviera dándola de manera presencial. Además el docente debe tener en claro que el uso de una plataforma virtual no será suficiente para realizar las prácticas de enseñanza-aprendizaje, en palabras de Carpio Vera et al., (2019),

“... deben poseer ineludiblemente competencias digitales, estas deberían estar relacionadas con la búsqueda de información, la comunicación y colaboración, la creación de contenidos, la seguridad 
y la resolución de problema ... se necesita que el docente tenga la motivación necesaria, considerando que la relación de estos dos actores, se produce de manera síncrona y asíncrona." (p.57).

\section{CONCLUSIÓN}

La comunicación en la educación es indispensable debido a su utilidad durante el intercambio de información, esto para que el alumnado pueda aprender y entender todo aquello que el profesor o docente está queriendo enseñar. La llegada de las Tecnologías de Información y Comunicación fue el comienzo de una nueva era de comunicación, posibilitando la transmisión de información a largas distancias, si uno quiere puede subir cualquier documento o archivo multimedia a la internet para que otras personas de diferentes partes del mundo puedan verlo, todo esto desde la comodidad de un equipo de cómputo o en un teléfono inteligente, un poder de comunicación sin límite que apareció en el mundo y que sufrió un gran cambio con su llegada.

Ecuador, siendo un país que ligeramente va avanzando a una velocidad menor que muchos otros países más estables, ha demostrado que cuando ve una oportunidad, no pierde el tiempo en aprovecharla, así con la existencia de las TIC, se decidió por darles un uso en el ámbito educativo como un apoyo para el docente, con esto se reducen diversas cargas que ha tenido en los últimos años, muchas de esas pueden ser la comunicación con aquellos alumnos que viven en lugares lejanos y la única manera para charlar con ellos es solo de forma presencial, también a la hora de investigar futuros temas a tratar a los estudiantes, el internet da la posibilidad de buscar cualquier tema simplemente introduciendo una palabra clave, también pueden compartir toda información encontrada con sus alumnos a través de una aplicación de mensajería instantánea o correo electrónico. Hasta dando la posibilidad de entablar una clase completa a través de videoconferencia, en la actualidad esto es el método de enseñanza que más se está usando debido a la situación mundial con el virus del COVID-19, limitando las posibilidades de asistir de manera presencial.

Todas las conclusiones fueron realizadas gracias a la continua investigación bibliográfica con su respectivo análisis, varias de estos demostraron diversos aspectos que ayudaron a completar el presente articulo y complemente de mejor manera al tema propuesto. Varios valores estadísticos presentados en la investigación fueron conseguidos gracias a las tesis encontradas, cuyos resultados han sido de gran ayuda a lo largo del presente documento. Una gran cantidad de autores ven a las TIC como las herramientas del futuro educacional, donde ya no será necesaria el hacer acto de presencia dentro de un aula, y la transferencia de información a través de la internet es constantemente utilizada, llegando al punto de completar una carrera universitaria completa sin haber puesto un pie dentro de la institución de educación 


\section{REFERENCIAS}

Acosta, T., \& Luján-Mora, S. (2017). Análisis de la accesibilidad de los sitios web de las universidades ecuatorianas de excelencia. Enfoque UTE, 8(1), 46-61. https://doi.org/10.29019/enfoqueute.v8n1.133

AGUIAR, B. O., VELÁZQUEZ, R. M., \& AGUIAR, J. L. (2019). Innovación docente y empleo de las TIC en la Educación Superior. Revista ESPACIOS, 40(02).

Bustos González, A., \& Fernández Porcel, A. (2020). Directrices para la creación de repositorios institucionales en universidades y organizaciones de educación superior. http://biblioteca.ucv.clhttp//agora.ucv.clhttp://www.ugr.es/ bilio/http://www.ugr.es/ afporcel/repositori o1.htm/http://europa.eu.int/comm/europeaid/projects/alfa

Carpio Vera, D. A., Paguay Balladares, W. J., \& Intriago Alcívar, G. C. (2019). LAS TIC EN LA PRÁCTICA DOCENTE UNIVERSITARIA BAJO LA MODALIDAD DE ESTUDIO VIRTUAL. Magazine de Las Ciencias: Revista de Investigación e Innovación. https://revistas.utb.edu.ec/index.php/magazine/article/view/622

Cortés, F. R. (2017). Uso de Google for education en los departamentos de orientación: colaborando para la mejora de la orientación y la atención a la diversidad. Revista AOSMA, (23), 80-84.

Domínguez Dias, Y. (2016). La utilización de las Tecnologías de la Información y las Comunicaciones (TIC) en el aprendizaje universitario. Universidad y Sociedad [seriada en línea]. Revista Universidad $Y$ Sociedad, 8(4), 158-163.

Espinoza Freire, E. E., Jaramillo Martinez, M., Cun Jaramillo, J., \& Pambi Encalada, R. (2018). La implementación de las TIC en el proceso de enseñanza-aprendizaje. Revista Metropolitana de Ciencias Aplicadas, 1(3), 10-17. http://remca.umet.edu.ec/index.php/REMCA/article/view/46

Falconí Asanza, A. V. (2017). Inclusión de la tecnología móvil de información y comunicación educativa como estrategia pedagógica. Universidad y Sociedad, 9(4), 82-89. http://rus.ucf.edu.cu/index.php/rus

Felipe, E., Maldonado, N., Orlando, M., Galarza, E., Ramiro, J., \& Jirón, G. (2017). Influencia de las TIC en la educación universitaria, caso Universidad Técnica de Machala. INNOVA Research Journal, 2(3), 69-79. https://dialnet.unirioja.es/servlet/articulo?codigo=5920526\&info=resumen\&idioma=SPA

Fernando Martínez-Sarmiento, L., Leticia, M., \& González, G. (2019). Utilización de la plataforma virtual Moodle para el desarrollo del aprendizaje autorregulado en estudiantes universitarios. Educar, 55(2), 479498. https://doi.org/10.5565/rev/educar.883

Haro, Y. R. (2020). Implementación de las TIC's en el aula con el uso de la herramienta "Google for education. ” Universitat Jaume I. http://repositori.uji.es/xmlui/handle/10234/190160

Hernández, R. M., Orrego Cumpa, R., \& Quiñones Rodríguez, S. (2018). Nuevas formas de aprender: La formación docente frente al uso de las TIC. Propósitos y Representaciones, 6(2), 671. https://doi.org/10.20511/pyr2018.v6n2.248

Huaman-Huayllapuma, M. (2019). E-learning y Aprendizaje Significativo en los Estudiantes de la Escuela Profesional de Historia de la Universidad Nacional de San Antonio Abad del Cusco. In Universidad Nacional de Educación Enrique Guzmán y Valle. Universidad Nacional de Educación Enrique Guzmán 
y Valle. http://repositorio.une.edu.pe/handle/UNE/4637

Lenin Zambrano-Zambrano, N., Antonio Mawyin-Cevallos, F., Andrés Gutiérrez-Santana, J., Eugenia Intriago-Cedeño, M., \& Adolfo Santana-Sardi, G. (2021). Análisis de criterio sobre la tecnología de la información y comunicación como herramienta de apoyo en la comunicación educativa universitaria. Revista Científica FIPCAEC (Fomento de La Investigación y Publicación En Ciencias Administrativas, Económicas y Contables). ISSN : 2588-090X .

Polo de Capacitación, Investigación y Publicación (POCAIP), 6(23), 977-995. https://doi.org/10.23857/fipcaec.v6i1.384

Mayre Barceló Hidalgo, L., Mabelín Del Sol Moreno, L., Ana Valdés Portillo, L., Km, B., mayrebarcelo, C., \& mabelindelsol, C. (2016). LA COMUNICACIÓN EDUCATIVA EN TIEMPOS DE REVOLUCIÓN TECNOLÓGICA.

Mendieta, G. N., \& García Mendieta, C. R. (2018). LAS TIC Y LA EDUCACIÓN ECUATORIANA EN TIEMPOS DE INTERNET: BREVE ANÁLISIS. Espirales: Revista Multidisciplinaria de Investigación, 2(15). http://www.revistaespirales.com/index.php/es/article/view/220/167

Obregón, R. (2007). Comunicación, desarrollo y cambio social.

Palacios Gordillo, C. N., \& Vergara Tejada, E. V. (2017). Las técnicas e-learning en el rendimiento académico de los estudiantes para el aprendizaje de los números reales y expresiones algebraicas. Universidad de Guayaquil, Facultad de Filosofía, Letras y Ciencias de la Educación. http://repositorio.ug.edu.ec/handle/redug/24774

Río, C. J., Calle, R. C., Elena Martín Pastor, M., \& Robaina, N. F. (2018). Rendimiento académico en educación superior y su asociación con la participación activa en la plataforma Moodle. Estudios Sobre Educacion, 34, 177-198. https://doi.org/10.15581/004.34.177-198

Salinas, J. (2004). Cambios metodológicos con las TIC: estrategias didácticas y entornos virtuales de enseñanza-aprendizaje. Bordón. Revista de Pedagogía, 56(3), 469-481. https://www.researchgate.net/publication/39214325

Tarango, J., Machin-Mastromatteo, J. D., \& Romo-González, J. R. (2019). Evaluación según diseño y aprendizaje de Google Classroom y Chamilo. IE Revista de Investigación Educativa de La REDIECH, 10(19), 91-104. https://doi.org/10.33010/ie_rie_rediech.v10i19.518

Vinueza, S. F. V., \& Gallardo, V. P. S. (2017). Impacto de las TIC en la Educación Superior en el Ecuador. Revista Publicando, 4(11 (1)), 355-368.

Xiomira, A., \& Torres, J. (2019). PÁGINAS WEB EDUCATIVAS. Introducción, la Web en los ambientes educativos, educación y Web, ventajas y desventajas, diseño, tipos de información, herramientas para construir una página Web, aplicación. In Universidad Nacional de Educación Enrique Guzmán y Valle. Universidad Nacional de Educación Enrique Guzmán y Valle. http://repositorio.une.edu.pe/handle/UNE/3986

Zempoalteca Durán, B., Barragán López, J. F., González Martínez, J., \& Guzmán Flores, T. (2017). Teaching training in ICT and digital competences in Higher Education System. Apertura, 9(1), 80-96. https://doi.org/10.32870/ap.v9n1.922 